\title{
DESCOMPOSICION TERMICA DEL DIPEROXIDO DE PINACOLONA (3,6-DITERBUTIL-3,6-DIMETIL-1,2,4,5- TETRAOXACICLOHEXANO) EN SOLUCIÓN DE 2-METOXIETANOL
}

\author{
Gladys N. Eyler ", Adriana I. Cañizo e Rosa K. Nesprias \\ Facultad de Ingeniería, Universidad Nacional del Centro de la Provincia de Buenos Aires, Av. Del Valle 5737, (B7400JWI) \\ Olavarría, Buenos Aires - Argentina
}

Recebido em 5/1/01; aceito em 14/11/01

\begin{abstract}
THERMAL DECOMPOSITION OF PINACOLONE DIPEROXIDE (3,6-ditertbutyl-3,6-dimethyl-1,2,4,5-tetraoxacyclohexane) IN 2-METHOXYETHANOL SOLUTION. The thermal decomposition reaction of pinacolone diperoxide (DPP; $0.02 \mathrm{~mol} \mathrm{~kg}^{-1}$ ) in 2methoxyethanol solution studied in the temperature range of $110.0-150.0{ }^{\circ} \mathrm{C}$, follows a first-order kinetic law up to at least $50 \%$ DPP conversion. The organic products observed were pinacolone, methane and tert-butane. A stepwise mechanism of decomposition was proposed where the first step is the homolytic unimolecular rupture of the $\mathrm{O}-\mathrm{O}$ bond. The activation enthalpy and activation entropy for DPP in 2-methoxyethanol were calculated $\left(\Delta \mathrm{H}^{\#}=43.8 \pm 1.0 \mathrm{kcal} \mathrm{mol}^{-1}\right.$ and $\left.\Delta \mathrm{S}^{\#}=31.9 \pm 2.6 \mathrm{cal} \mathrm{mol}^{-1} \mathrm{~K}^{-1}\right)$ and compared with those obtained in other solvents to evaluate the solvent effect.
\end{abstract}

Keywords: cyclic peroxide; kinetic; solvent effect.

\section{INTRODUCCION}

Los peróxidos orgánicos son compuestos que tienen gran importancia en numerosas reacciones de combustión e importantes aplicaciones industriales tales como iniciadores de polimerización, agentes de entrecruzamiento, blanqueadores, desinfectantes, etc.

Los estudios cinéticos y mecanísticos correspondientes a la termólisis en solución de peróxidos orgánicos cíclicos y todas las comparaciones entre ellos que de allí surgen, han sido el objetivo inicial de este grupo de investigación. Sin embargo, en los últimos años ha resultado de gran interés la aplicación de este tipo de compuestos como iniciadores polifuncionales en los procesos de polimerización radicálica de estireno. Si bien el diperóxido de pinacolona (3,6-diterbutil-3,6-dimetil-1,2,4,5-tetraoxaciclohexano, DPP; Figura 1) fue utilizado hace varias décadas como iniciador en la obtención de poliestireno ${ }^{1}$, actualmente, a través de la introducción de algunas modificaciones en las condiciones de trabajo, se ha logrado su utilización a temperaturas relativamente altas y con mayores conversiones del monómero $(\mathrm{ca} .99 \%)^{2}$. Por lo tanto, el estudio del comportamiento de estos peróxidos en solución es complementario de los estudios relacionados con su aplicación en procesos industriales.<smiles>CC(C)(C)OOC(C)(C)OOC(C)(C)C</smiles>

Figura 1
Las técnicas de polimerización que se están desarrollando contemplan el agregado del peróxido cíclico polifuncional en estado sólido ${ }^{2}$ o en solución ${ }^{3}$, dando en cada caso diferentes resultados. De todos los peróxidos orgánicos cíclicos estudiados hasta el momento, el DPP y el triperóxido cíclico de dietilcetona (TPDEC) son los que han manifestado el mejor comportamiento como iniciadores polifuncionales. Sin embargo, el efecto de solvente que presentan algunos peróxidos orgánicos en su descomposición térmica no siempre se transmite en el mismo sentido en relación a su función como iniciador cuando se lo agrega en solución, como se ha evidenciado para el TPDEC 3 .

De acuerdo a los resultados obtenidos en estudios anteriores la constante de velocidad correspondiente a la descomposición térmica de DPP no es sensiblemente afectada cuando la reacción se lleva a cabo en benceno $^{4}$, acetonitrilo ${ }^{5}$, ácido acético ${ }^{5}$, n-octano $^{5}$ o tolueno ${ }^{6}$. Por ello, resulta de interés encontrar algún solvente donde se aumente la velocidad de la reacción de descomposición del DPP. En estudios anteriores con otros compuestos peroxídicos se ha determinado que cuando se utiliza como solvente 2-propanol ${ }^{7,8}$, ácido acético ${ }^{5,9}$ y 2-metoxietanol ${ }^{10,11}$ la descomposición de los peróxidos se acelera. El objetivo de este trabajo es comprobar si la termólisis del DPP en solventes de la familia de los alcoholes presenta algún comportamiento similar al observado para otros peróxidos cíclicos derivados de cetonas. En este trabajo se presentan los parámetros cinéticos y de activación del DPP en 2-metoxietanol, y se establecen comparaciones y correlaciones con los datos correspondientes obtenidos previamente para la misma reacción en otros solventes. Los resultados obtenidos serán utilizados para continuar con los estudios relacionados con el empleo de DPP como iniciador bifuncional en la polimerización de estireno.

\section{PARTE EXPERIMENTAL}

\section{Síntesis del diperóxido de pinacolona}

El diperóxido de pinacolona se prepara utilizando una modificación de la técnica descripta por $\mathrm{McCullough}^{12}$ para la obtención de diperóxidos cíclicos derivados de cetonas lográndose una mejora en el rendimiento de casi un 100 por ciento.

\footnotetext{
*e-mail: neyler@fio.unicen.edu.ar
} 
En un erlenmeyer enfriado a $-15{ }^{\circ} \mathrm{C}$ se colocan $14 \mathrm{~g}$ de ácido sulfúrico $70 \%$ (vol), sobre los cuales se agregan $6 \mathrm{~mL}$ de pinacolona $(0,048$ moles $)$, manteniendo el sistema en agitación constante. A esta mezcla, que se torna amarillenta, se le adicionan gota a gota desde una ampolla de decantación y en el transcurso de una hora y media $5,7 \mathrm{~mL}$ de peróxido de hidrógeno al $30 \%$ (0,056 moles). El precipitado blanco obtenido se filtra y se lava con agua destilada hasta eliminar el exceso de ácido, finalmente se lava con un pequeño volumen ( $2 \mathrm{~mL})$ de acetona y se coloca en el desecador $(3,23 \mathrm{~g}, 67 \%$ de rendimiento).

El producto crudo se purifica por recristalización (dos veces) con metanol (p.f: $122-124^{\circ} \mathrm{C}$, lit. ${ }^{12} 124^{\circ} \mathrm{C}$ ). Las bandas de absorción obtenidas por espectroscopía FTIR (KBr) son: 1)- 2988,183 (s) cm ${ }_{1}^{1}$ 2)-2970,215 (s) $\mathrm{cm}^{-1}$ 3)- 2927,200 (m) $\mathrm{cm}^{-1}$ 4)- 2878,032 (m) $\mathrm{cm}^{-1}$ 5)- $1485,423(\mathrm{~m}) \mathrm{cm}^{-1}$ 6)- $1459,500(\mathrm{~s}) \mathrm{cm}^{-1}$ 7)- $1390,500(\mathrm{~s}) \mathrm{cm}^{-1}$ 8)1124,637 (vs) $\mathrm{cm}^{-1}$ 9)- 1175,305 (s) $\mathrm{cm}^{-1}$ 10)- 1024,600 (m) $\mathrm{cm}^{-1}$ 11)- $939,249(\mathrm{~m}) \mathrm{cm}^{-1}$ 12)- 902,817 (s) $\mathrm{cm}^{-1}$ 13)- $815,200(\mathrm{~m}) \mathrm{cm}^{-1}$ 14)- $611,853(\mathrm{~m}) \mathrm{cm}^{-1}$ 15)- $546,953(\mathrm{~m}) \mathrm{cm}^{-1}$. Estos valores coinciden con los informados en la literatura ${ }^{12}$.

\section{Solventes utilizados}

El 2-metoxietanol (libre de peróxidos) utilizado como solvente y el n-octano (patrón interno) fueron purificados según técnicas apropiadas ${ }^{13}$ y controlada su pureza por CG.

\section{Determinaciones cinéticas}

Tubos Pyrex cerrados en un extremo ( $8 \mathrm{~cm}$ de largo x $2 \mathrm{~mm}$ diám. int.) fueron cargados con ca. $0,5 \mathrm{~mL}$ de la solución de DPP en el solvente adecuado, algunos sellados en presencia de oxígeno con la llama de un soplete y otros convenientemente degasificados en la línea de vacío $\left(-196^{\circ} \mathrm{C}\right)$ y cerrados de igual forma.

Estas ampollas fueron sumergidas en un baño de aceite de silicona termostatizado $\left( \pm 0,1^{\circ} \mathrm{C}\right)$ a la temperatura elegida, extrayéndose las mismas a tiempos convenientemente determinados y deteniendo la descomposición del DPP a $0{ }^{\circ} \mathrm{C}$, en un baño de hielo y agua.

Las determinaciones cuantitativas del DPP remanente en las soluciones pirolizadas, la cuantificación de la pinacolona y la determinación cualitativa de otros productos formados (metano, terbutano), se realizaron por CG utilizando el método de patrón interno (n-octano). Se empleó un cromatógrafo de gases marca Konik, modelo KNK 2000, equipado con detector FID, columna rellena tipo SE30 (metil silicona Gum-Rubber) y se utilizó nitrógeno como gas portador.

Los análisis se realizaron a temperatura programada manteniendo inicialmente la columna a $60^{\circ} \mathrm{C}$ durante $12 \mathrm{~min}$, luego se incrementó la temperatura a razón de $10{ }^{\circ} \mathrm{C}$ por minuto hasta alcanzar una temperatura final de $160{ }^{\circ} \mathrm{C}$. La temperatura del inyector se fijó en $125^{\circ} \mathrm{C}$.

Los valores de $\mathrm{k}_{\exp }$ de primer orden fueron calculados por el método de los mínimos cuadrados como la pendiente de la recta determinada cuando se representa ln [DPP] en función del tiempo.

Utilizando la ecuación de Arrhenius se analizó el efecto de la temperatura sobre la $\mathrm{k}_{\text {exp }}$ y se calculó la energía de activación y el factor preexponencial correspondiente. Los parámetros de activación $\left(\Delta \mathrm{H}^{\#} \mathrm{y} \Delta \mathrm{S}^{\#}\right)$ se calcularon utilizando la representación de Eyring.

\section{RESULTADOS Y DISCUSION}

La descomposición del DPP se ha estudiado en solución de 2metoxietanol, en el ámbito de temperaturas de $110,0-150,0^{\circ} \mathrm{C}$ y a concentraciones iniciales de $0,02 \mathrm{~mol} \mathrm{~kg}^{-1}$. La termólisis del DPP en 2-metoxietanol, cumple con una ley cinética de primer orden hasta conversiones del peróxido de $c a$. 50 por ciento. Si bien a concentraciones bajas del DPP los efectos de reacciones secundarias de descomposición inducida por radicales libres originados en el medio de reacción, pueden considerarse mínimos o despreciables, se realizaron algunas experiencias en presencia de oxígeno. Los resultados obtenidos indican que prácticamente no existen diferencias en los valores de las constantes de velocidad (Tabla 1) cuando el oxígeno está presente o cuando fue eliminado del medio, lo que indicaría que los radicales libres generados no inducen la descomposición del DPP.

A los efectos de corroborar que las constantes de velocidad calculadas no incluyen una contribución de reacciones inducidas por especies radicalarias derivadas del peróxido, se investigó el efecto de la concentración de DPP sobre el valor de la $\mathrm{k}_{\exp }$ a $140^{\circ} \mathrm{C}$ (Tabla 1). A causa de la baja solubilidad del DPP en 2-metoxietanol, la

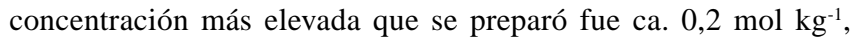
observándose que no existe efecto de la concentración sobre la velocidad de reacción.

Tabla 1. Constantes de velocidad de primer orden a distintas temperaturas correspondientes a la descomposición térmica de DPP en 2-metoxietanol

\begin{tabular}{ccc}
\hline Temperatura $\left[{ }^{\circ} \mathrm{C}\right]$ & $\begin{array}{c}\text { Concentración } \\
\text { inicial de DPP } \\
{\left[\mathrm{mol} \mathrm{kg}^{-1}\right]}\end{array}$ & $\begin{array}{c}10^{5} \mathrm{x} \mathrm{k}_{\text {exp }} \\
{\left[\mathrm{s}^{-1}\right]}\end{array}$ \\
\hline 110,0 & 0,021 & 0,7 \\
120,0 & 0,021 & 3,51 \\
130,0 & 0,021 & $3,42^{\mathrm{a}}$ \\
140,0 & 0,019 & 11,0 \\
& 0,019 & 75,8 \\
& 0,022 & 69,4 \\
150,0 & 0,019 & $75,5^{\mathrm{a}}$ \\
& 0,200 & 76,4 \\
& 0,022 & 137 \\
& 0,022 & $133^{\mathrm{a}}$ \\
\hline
\end{tabular}

${ }^{a}$ En presencia de oxígeno.

El efecto de la temperatura sobre los valores de $\mathrm{k}_{\text {exp }}$ (Tabla 1), puede representarse por la ecuación de Arrhenius (ec. 1), donde los errores mostrados corresponden a los desvíos estándar obtenidos por el tratamiento de los datos cinéticos con el método de los mínimos cuadrados $^{14,15}$ y la energía de activación está expresada en cal mol $^{-1}$.

$\ln \mathrm{k}_{\exp }\left[\mathrm{s}^{-1}\right]=(46,8 \pm 2,6)-(44650 \pm 1038) / \mathrm{RT}$

La linealidad de la ecuación de Arrhenius $(\mathrm{r}=0,990)$ en un intervalo de temperaturas relativamente amplio $\left(40^{\circ} \mathrm{C}\right)$, sugiere que los parámetros de activación calculados (energía de activación y factor preexponencial, ec. 1), para la reacción del DPP en 2-metoxietanol corresponden a un proceso simple representado por la ruptura homolítica $^{16,17}$ del enlace $\mathrm{O}-\mathrm{O}$, (ec. 2):

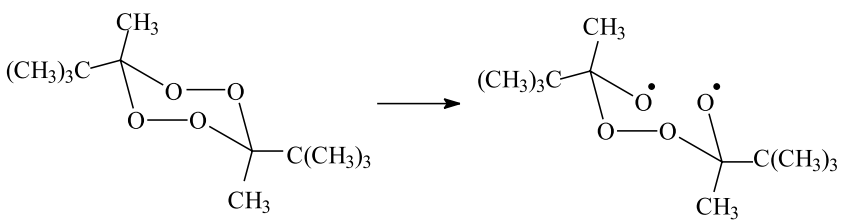


El birradical intermediario (ec. 2), puede experimentar posteriores reacciones que involucren rupturas de enlaces $\mathrm{C}-\mathrm{O}$, justificando la aparición de pinacolona con un rendimiento del $60 \%(1,2 \mathrm{~mol}$ pinacolona/mol DPP descompuesto), o rupturas C-C dando lugar a radicales terbutilo y metilo, que luego reaccionan con el solvente extrayendo hidrógeno para formar metano (ec. 3) y terbutano (ec. 4):

$$
\begin{aligned}
& \mathrm{CH}_{3}^{\cdot}+\mathrm{RH} \stackrel{-\mathrm{R}^{\cdot}}{\rightarrow} \mathrm{CH}_{4} \\
& \left(\mathrm{CH}_{3}\right)_{3} \mathrm{C}^{\cdot}+\mathrm{RH} \stackrel{-\mathrm{R}^{\cdot}}{\rightarrow}\left(\mathrm{CH}_{3}\right)_{3} \mathrm{CH}
\end{aligned}
$$

Los radicales $\mathrm{R} \cdot$ que derivan del solvente vuelven a reaccionar con él dando una serie de compuestos de elevado peso molecular entre los cuales se pueden mencionar el 2,5,8,11,14- pentaoxapentadecano y el 2,5,8,11,14,17-hexaoxaoctadecano, detectados por CG/EM.

La termólisis de algunos tetraoxaciclohexanos sustituídos indica que el rendimiento de la correspondiente cetona y de los otros productos de reacción depende fuertemente del tipo de sustrato ${ }^{18} \mathrm{y}$ de la naturaleza del solvente de reacción ${ }^{19}$.

El efecto del solvente sobre la $\mathrm{k}_{\text {exp }}$ puede analizarse a través de la representación del logaritmo natural de la constante de velocidad para la descomposición del DPP en distintos solventes en función de algún parámetro empírico de polaridad de los mismos. En la Figura 2 se presenta la correlación existente entre el logaritmo natural de las constantes de velocidad y el parámetro empírico de polaridad del solvente $\mathrm{E}_{\mathrm{T}}(30)$ determinado por Dimroth y Reichardt ${ }^{20-22}$ para una importante cantidad de solventes y mezclas de solventes. Este parámetro se basa en la energía de transición, medida en $\mathrm{kcal} \mathrm{mol}^{-1}$, asociada a la banda de absorción solvatocrómica de mayor longitud de onda del colorante betaína piridinio $\mathrm{N}$-fenóxido (colorante $\mathrm{n}^{\circ} 30$ en el trabajo original de dichos autore ${ }^{20}$ ) o de algún otro derivado de la betaína. Este parámetro es una medida de las interacciones entre soluto/solvente en la región cibotáctica y no tiene equivalencia directa con otras propiedades macroscópicas del solvente como la constante dieléctrica o el momento dipolar. Al respecto, puede observarse que a medida que aumenta la polaridad del solvente, determinada a través del parámetro $\mathrm{E}_{\mathrm{T}}(30)$, también aumenta la velocidad de descomposición del peróxido, sin embargo esta correlación no es lineal. Este hecho nos permite sugerir que las interacciones específicas soluto/solvente son condicionantes de la reactividad del DPP en solución. Se ha demostrado en la bibliografía ${ }^{5,7-9}$ que para diferentes peróxidos cíclicos estas interacciones corresponden a enlace puente de hidrógeno entre el solvente prótico y el enlace O-O.

Por otro lado, si se analizan los parámetros de activación, puede observarse que los correspondientes a las determinaciones cinéticas en 2-metoxietanol (Tabla 2) son muy superiores a aquellos observados en los otros solventes. El elevado valor hallado para la entropía

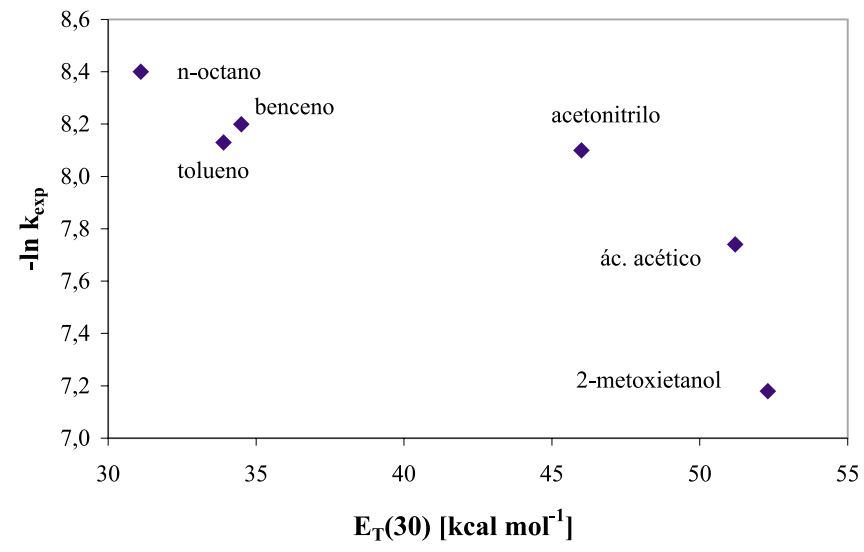

Figura 2. Representación gráfica del ln $k_{\text {exp }}$ versus el parámetro de polaridad del solvente $E_{T}(30)$, correspondiente a la descomposición térmica de DPP en diferentes solventes, a $140{ }^{\circ} \mathrm{C}$

de activación en 2-metoxietanol es comparable al informado ${ }^{9}$ para la descomposición térmica de una trioxazina sustituída en ácido acético.

En general, en una serie de reacciones ${ }^{23}$ que involucre cambios en el solvente o moderados cambios en la estructura de los compuestos considerados, la entalpía y la entropía varían, pero no en forma independiente. En este caso, se observa la existencia de una compensación entre los parámetros de activación ya que se mantiene la constancia en los valores de la energía libre de activación en los diferentes solventes. Sin embargo, cabe aclarar que cuando en una serie de reacciones se estudian efectos de solvente sobre la velocidad de reacción, no resulta suficiente a fin de establecer conclusiones considerar únicamente el cambio en la energía libre de activación correspondiente, ya que la misma está determinada por las contribuciones de los términos entálpico y entrópico. A veces la correlación entre ambos parámetros se aproxima a una relación lineal característica para esa serie de reacciones en particular ${ }^{24}$. Existen en la literatura ${ }^{4-6,8,9,25}$ antecedentes que muestran este efecto para otros peróxidos cíclicos.

La existencia de una compensación lineal entre los parámetros de activación para la descomposición térmica del DPP en distintos solventes (Tabla 2) de acuerdo con el criterio de Leffler ${ }^{24}$, también conocida como "efecto de compensación", puede representarse a través de la ecuación (5), que muestra una relación aproximadamente lineal $(\mathrm{r}=0,976)$, siendo $426 \mathrm{~K}\left(153^{\circ} \mathrm{C}\right)$ el valor de la "temperatura isocinética" $(\beta)$, o sea la temperatura a la cual en todos los solventes la reacción de descomposición térmica de DPP transcurriría a la misma velocidad.

$\Delta \mathrm{H}^{\#}=\Delta \mathrm{H}^{0}+\beta \Delta \mathrm{S}^{\#}$

Tabla 2. Valores de los parámetros de activación y constantes de velocidad $\left(\mathrm{k}_{\mathrm{exp}}\right)$ correspondientes a la descomposición térmica a $140{ }^{\circ} \mathrm{C}$ del DPP en diferentes solventes

\begin{tabular}{lccccc}
\hline Solvente & $10^{5} \mathrm{x} \mathrm{k}_{\exp }\left[\mathrm{s}^{-1}\right]$ & $\Delta \mathrm{H}^{\#}\left[\mathrm{kcal} \mathrm{mol}^{-1}\right]^{\mathrm{a}}$ & $\Delta \mathrm{S}^{\#}\left[\mathrm{cal} \mathrm{mol}^{-1} \mathrm{~K}^{-1}\right]^{\mathrm{a}}$ & $\Delta \mathrm{G}^{\#}\left[\mathrm{kcal} \mathrm{mol}^{-1}\right]^{\mathrm{a}}$ & $\mathrm{Referencia}$ \\
\hline Benceno & 27,4 & $29,8 \pm 0,6$ & $-1,5 \pm 1,5$ & $30,4 \pm 0,6$ & 4 \\
Ac. Acético & 43,4 & $32,2 \pm 1,4$ & $5,3 \pm 3,6$ & $30,0 \pm 1,4$ & 5 \\
2-propanol/benc. & $63,0^{\mathrm{b}}$ & $33,7 \pm 1,4$ & $10,7 \pm 3,0$ & $29,3 \pm 1,4$ & 6 \\
Acetonitrilo & 30,5 & $34,6 \pm 0,6$ & $10,8 \pm 1,5$ & $30,1 \pm 0,6$ & 5 \\
n-octano & 23,2 & $37,5 \pm 0,2$ & $12,3 \pm 0,5$ & $32,4 \pm 0,2$ & 5 \\
2-metoxietanol & 75,8 & $43,8 \pm 1,0$ & $31,9 \pm 2,6$ & $30,7 \pm 1,0$ & Este trabajo \\
Tolueno & 29,6 & $34,6 \pm 1,2$ & $8,8 \pm 3,0$ & $31,0 \pm 1,2$ & 5 \\
\hline
\end{tabular}

a errores calculados como en referencia $15,{ }^{\mathrm{b}}$ valor de $\mathrm{k}_{\text {exp }}$ calculado por extrapolación 
Dado que los estudios cinéticos del DPP en cada solvente se han realizado en un intervalo de temperaturas de al menos $30^{\circ} \mathrm{C}$ y que los errores en los parámetros de activación (Tabla 2) son despreciables frente a las diferencias observadas entre los mismos $\left(\Delta \Delta \mathrm{H}^{\#} \cong\right.$ $14 \mathrm{kcal} \mathrm{mol}^{-1}$ y $\Delta \Delta \mathrm{S}^{\#} \cong 33,4 \mathrm{cal} \mathrm{mol}^{-1} \mathrm{~K}^{-1}$ ), podría considerarse que la correlación de acuerdo al criterio de Leffler ${ }^{12}$ es válida. Sin embargo al analizar la reactividad del DPP en varios solventes a distintas temperaturas, el peróxido muestra comportamientos diferentes, por ejemplo a $140{ }^{\circ} \mathrm{C}$ (Tabla 2) la reacción es más rápida en solventes próticos, como 2-propanol o 2-metoxietanol, que en solventes no próticos.

Estas observaciones concuerdan con el hecho de que la relación isocinética ha sido muy criticada ${ }^{26-28}$ cuando se utiliza como único tratamiento para analizar la existencia de efecto de solvente o de sustituyentes sobre una reacción dada y su validez debe ser consistente con el tratamiento propuesto posteriormente por Exner ${ }^{26}$. Así la representación del logaritmo natural de la constante de velocidad en cada solvente en función de 1/T (Figura 3) debe ser lineal de acuerdo con la ecuación de Arrhenius y dichas líneas deben interceptarse en un punto que se conoce como la inversa de la "temperatura isocinética" $\left(\beta^{-1}\right)$. La aplicación de un método estadístico ${ }^{27,28}$ permitiría obtener la función $\mathrm{S}_{\mathrm{u}}$ (suma residual de cuadrados) cuyo mínimo corresponde a $\beta^{-1}$. Para este caso en particular, se observa que no existe dicho punto de intersección ya que las rectas se cortan de a pares en todo el rango de temperaturas experimentales, por lo cual no es posible aplicar el tratamiento estadístico para determinar el valor de $\beta$. Además, de acuerdo al tratamiento de Leffler, la temperatura isocinética cae dentro del rango de temperaturas experimentales, por lo cual no resulta sorprendente encontrar inversión en el comportamiento cinético de los diferentes sistemas estudiados a las distintas temperaturas consideradas. Sin embargo, es evidente que la reacción de descomposición térmica del DPP es afectada por solventes de diferentes características fisicoquímicas (Tabla 2). Por ello, se postula que en la serie de reacciones consideradas, donde el mecanismo de reacción es común y corresponde a la ruptura homolítica unimolecular del enlace $\mathrm{O}-\mathrm{O}$, se ponen de manifiesto diferentes mecanismos de interacción soluto /solvente, a los cuales pueden atribuirse los cambios en los valores de los parámetros de activación.

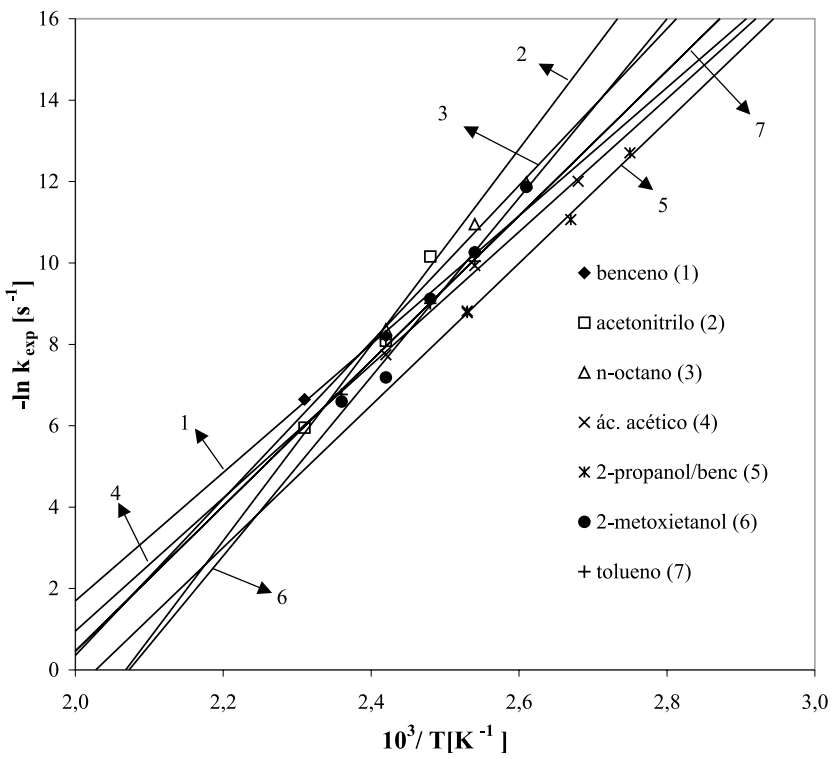

Figura 3. Representación gráfica de la ecuación de Arrhenius correspondiente a la descomposición térmica del DPP en diferentes solventes

\section{CONCLUSIONES}

1 - La termólisis del DPP en 2-metoxietanol sigue una cinética de primer orden hasta al menos una vida media.

2 - Las condiciones de trabajo elegidas permiten descartar reacciones secundarias inducidas por radicales libres derivados del peróxido por lo cual los valores de $\mathrm{k}_{\exp }$ informados corresponden a la ruptura homolítica del enlace peroxídico.

3 - El efecto observado en la reactividad del DPP a diferentes temperaturas y en diferentes solventes es de gran utilidad al momento de utilizar estos sistemas para la obtención de polímeros derivados de estireno.

\section{REFERENCIAS}

1. Walter, H. A.; US pat. 2591645 1952. ( $C A$ 1953, 47, 601).

2. Cerna, J. R.; Morales, G.; Eyler, G. N.; Cañizo, A. I; J. Appl. Polym. Sci. 2002, 83, 1 .

3. Cerna, J. R.; Morales, G.; Cañizo, A. I.; Eyler, G. N.; J. Org. Chem. 2001, submetido.

4. Cafferata, L. F. R.; Eyler, G. N.; Cañizo, A. I.; Svartman, E. L.; Borkowski, E. J.; J. Org. Chem. 1990, 55, 1058.

5. Cafferata, L. F. R.; Eyler, G. N.; Svartman, E. L.; Cañizo, A. I. ; Alvarez, E. E.; J. Org. Chem. 1991, 56, 411.

6. Eyler, G.N.; Cañizo, A.I.; trabalho no publicado.

7. Cañizo, A. I.; Cafferata, L. F. R.; An. Asoc. Quim. Argent. 1992, 80, 345.

8. Eyler, G. N.; Cañizo, A. I.; Alvarez, E. E.; Cafferata, L. F. R.; An. Asoc. Quim. Argent. 1994, 82, 175; Eyler, G. N.; Tesis de Maestría; UNER, Argentina, 1996.

9. Cafferata, L. F. R.; Nojima M.; Yamakoshi H.; Int. J. Chem. Kim. 1996, 28, 21; Eyler, G. N.; Cañizo, A. I.; Alvarez, E. E.; Revista Copaqui 1995, $15,953$.

10. Leiva, L.; Gómez Vara, E.; trabalho no publicado.

11. Eyler, G. N.; trabalho no publicado.

12. McCullough, K. J.; Morgan, A. R.; Nonhebel, D. C.; Pauson, P. L.; White, G. J.; J. Chem. Res., Synop. 1980, 34, M 0601.

13. Perrin, D. D.; Armarego, W. L. F.; Purification of Laboratory Chemicals, Pergamon Press, $3^{\circ}$ ed., 1988, p 218.

14. Schaleger, L. L.; Long, F. A.; Adv. Phys. Org. Chem. 1963, $1,1$.

15. Huyberechts, S.; Halleux, A.; Kruys, P.; P. Bull, Soc. Chim. Belg. 1955, 64, 203.

16. Cafferata, L.F.R.; Eyler, G.N.; Mirífico, M.V.; J. Org. Chem. 1984, 49, 2107.

17. Castro, E. A.; Villar, H.O.; Stradella, O. G.; J. Mol. Struct. Theochem. 1986, 7, 135.

18. McCullough, K. J.; Morgan, A. R.; Nonhebel, D. C.; Pauson, P. L.; J. Chem. Res., Synop. 1981, 35, M 0629. Ibid 36, M 0651.

19. Sanderson, J. R.; Story, P. R.; J. Org. Chem. 1974, 39, 3463.

20. Dimroth, K.; Reichardt, C.; Siepmann, T.; Bohlmann, F.; Liebigs Ann. Chem. 1963, 661, 1 .

21. Dimroth, K.; Reichardt, C.; Siepmann, T.; Bohlmann, F.; Liebigs Ann. Chem. 1969, 93, 727.

22. Reichardt, C. En Solvents and Solvent Effects in Organic Chemistry, $2^{\circ}$ ed. (Suiza), Cambridge; New York, NY: VCH, 1988, cap. 7, p. 359.

23. Bunnett, J. F. En Investigation of Rates and Mechanisms of Reaction, Parte I, vol VI de Techniques of Chemistry, Weissberger, A. ed., Wiley: NY, 1974, Cap. VIII, p 367.

24. Leffler, J. E.; J. Org. Chem. 1955, 20, 1202.

25. Eyler, G. N.; Cañizo, A. I.; Mateo, C. M.; Alvarez, E. E.; Cafferata, L. F. R.; J. Org. Chem. 1999, 64, 8457.

26. Exner, O.; Collect. Czech. Chem. Commun. 1972, 37, 1425

27. Linert, W.; Jameson, R. F.; Chem. Soc. Rev. 1989, 18, 477.

28. Petersen, R. C.; J. Org. Chem. 1964, 29, 3133. 